\title{
High-sensitivity troponin is associated with high risk clinical profile and outcome in acute heart failure
}

\author{
Mirta Diez ${ }^{1}$, María Luján Talavera ${ }^{1}$, Diego Gabriel Conde ${ }^{2}$, \\ Roberto Campos ${ }^{1}$, Adriana Acosta ${ }^{1}$, Marcelo Sergio Trivi ${ }^{3}$ \\ ${ }^{1}$ Heart Failure Section, Instituto Cardiovascular de Buenos Aires, Argentina \\ ${ }^{2}$ Coronary Care Unit, Instituto Cardiovascular de Buenos Aires, Argentina \\ ${ }^{3}$ Department of Cardiology, Instituto Cardiovascular de Buenos Aires, Argentina
}

\begin{abstract}
Background: The aim of the study was to evaluate the value of high-sensitivity cardiac troponin (hs-cTn) for identifying high-risk patients.

Methods and results: One hundred and eighty-seven patients admitted with acute heart failure (HF) (without myocardial infarction) were consecutively included; hs-cTn was measured at admission; the relation between elevated hs-cTn and the clinical outcome during hospitalization and at 90 days was analyzed; 93\% $(n=174)$ had hs-cTn above the maximal normal value (14 ng/L); median hs-cTn was $42 \mathrm{ng} / \mathrm{L}$ (IQR 24-81). Patients with ejection fraction $(E F) \leq 45 \%$ had higher hs-cTn values ( $p=0.0004)$. Patients with low cardiac output syndrome (LCOS) or shock had higher troponin levels compared with those with less severe clinical presentations $(p=0.004)$. Patients who required inotropic presented higher troponin values $(p=0.002)$, troponin values were also higher in those requiring complex therapies (intra-aortic balloon pump, mechanical ventilation or hemodialysis, $p=0.002)$. At 90-day follow-up, 28 (15.5\%) patients died and 27 rehospitalizations occurred (55 events). The risk of events was greater in patients with $h s-c T n>42 n g / L$ (0.021), low blood pressure at admission ( $p=0.002), L C O S$ or shock ( $p<0.0001), E F \leq 45 \%(p=0.005)$ and inotropic use $(p<0.0001)$. In multivariate analysis, only inotropic agents requirements was associated independently with a high risk of death or rehospitalizations at 90 days $(p=0.007)$.
\end{abstract}

Conclusions: Elevation of $h s-c T n$ is a finding almost constant in patients with decompensated HF. In subjects with higher troponin levels ventricular dysfunction is frequent. The use of hs-cTn for risk stratification at admission helps to identify populations with poor outcome during hospitalization and increased risk of death or rehospitalizations during follow-up who will require rapid implementation of aggressive treatment. (Cardiol J 2016; 23, 1: 78-83)

Key words: high-sensitivity cardiac troponins, decompensated heart failure, mortality (or outcome)

\section{Introduction}

The diagnostic role of high-sensitivity cardiac troponin (hs-cTn) in acute coronary syndromes (ACS) has been recently validated, and the useful- ness of this biomarker has also been evaluated in heart failure $(\mathrm{HF})$ populations $[1,2]$.

The initial evaluation of patients with acute HF should include the determination of the severity of the disease to indicate an early and appropriate

Address for correspondence: Dr María Luján Talavera, Staff Physician of the Heart Failure Section, Instituto Cardiovascular de Buenos Aires, Libertador AV 6302, Buenos Aires, Argentina, e-mail: mltalavera@icba.com.ar 
treatment, and the type of complexity of the hospitalization area. The value of hs-cTn for identifying high-risk patients has not been studied in depth and thus remains uncertain.

The goals of this study were: 1 ) to determine the prevalence of hs-cTn in patients with decompensated HF; 2) to study the association between high troponin levels and the clinical outcome during hospitalization, and 3) to evaluate the association between troponin levels and the clinical outcome at 90 days.

\section{Methods}

\section{Study population}

One hundred and eighty-seven patients admitted between January 2013 and March 2014 to the intensive care unit of the Instituto Cardiovascular de Buenos Aires with decompensated HF were consecutively included.

Patients $<18$ years old, those with ACS (defined by elevated cardiac enzymes associated with chest pain and/or electrocardiographic changes) or resuscitated from cardiac arrest were excluded.

The diagnosis of HF was based on the anamnesis, physical examination and the results of the complementary tests.

Ischemic etiology was defined as the presence of previous myocardial infarction ( $Q$ waves in two contiguous electrocardiographic leads), history of reperfusion (percutaneous coronary intervention or revascularization surgery) or coronary artery disease documented by coronary angiography (left main coronary artery stenosis $>50 \%$ or stenosis of other coronary vessel $>70 \%$ ).

A categorical variable was defined from the variable ejection fraction (EF) and patients were divided in two groups, based on a cutoff point of $45 \%$ : one with $\mathrm{EF}>45 \%$ and the other with $\mathrm{EF} \leq 45 \%$.

A categorical variable was defined from the variable hs-cTn, and patients were divided in two groups based on a cutoff point of $42 \mathrm{ng} / \mathrm{L}$ (median value observed): one with hs-cTn $>42 \mathrm{ng} / \mathrm{L}$ and the other with hs-cTn $\leq 42 \mathrm{ng} / \mathrm{L}$.

"Complex therapy" was defined of the necessity of any of the following treatments: intra-aortic balloon pump, mechanical ventilation or hemodialysis.

The patients were followed-up at 90 days after discharge by telephone calls or by revising the medical record. An event was defined as the occurrence of death and/or readmission due to decompensated HF within 90 days.

The protocol was conducted following the recommendations of the Declaration of Helsinki and all the patients signed an informed consent form. The protocol was approved by the Committee on Ethics of our institution.

\section{Troponin measurement}

Troponin was measured after the admission at the emergency room, using a commercially available kit (ECLIA Elecsys 2010 analyzer, Roche Diagnostics, Germany). A value $<14 \mathrm{ng} / \mathrm{L}$ was considered normal. Troponin values above the median of the sample were considered elevated.

\section{Statistical analysis}

Categorical variables were expressed as frequencies and percentages and continuous variables as means and standard deviation or medians with their corresponding 25-75\% interquartile range (IQR), as applicable.

The differences between the categorical variables were estimated using the $\chi^{2}$ test. The differences between the medians defined by the categorical variables were compared using the Wilcoxon test.

As the variable troponin was asymmetric and skewed to the right, with a wide range of values, logarithmic transformation was used to improve the visualization of the data, obtaining values between 1 and 6 , approximately. We also evaluated if logarithmic transformation (used in the references) could normalize the data. The odds ratio and hazard ratio (HR) were calculated with their corresponding $95 \%$ confidence interval (CI). The Rothman method was used to estimate the HR of the CI.

Logistic regression analysis was performed to determine the variables associated or not with the event at 90 days. The association between hs-cTn level and the probability of events at 90 days, and the association between each variable of interest with such probability were analyzed using univariate and multivariate Cox regression analyses. The validity of the assumption of Cox proportional hazards was analyzed with a global test and for each variable using adequate tests. The Cox-Mantel test was used to compare the survival curves between the different groups. Survival was estimated using the Kaplan-Meier method. Statistical analysis was performed using the software packages $\mathrm{R} v$. 3.0.1., SPSS v. 20, InfoStat v. 2014py, STATA v. 12IC.

\section{Results}

One hundred and eighty-seven patients admitted between January 2013 and March 2014 with decompensated HF were consecutively included. 
Table 1. Characteristics of the population.

\begin{tabular}{|c|c|}
\hline Characteristic & Value $(n=187)$ \\
\hline Age & $78(S D \pm 11)$ \\
\hline Male sex & $108(57.8 \%)$ \\
\hline Ischemic etiology & $87(46.5 \%)$ \\
\hline \multicolumn{2}{|l|}{ Cardiovascular risk factors: } \\
\hline Current smoker & $9(4.8 \%)$ \\
\hline Hypertension & $153(81.8 \%)$ \\
\hline Diabetes & $46(24.6 \%)$ \\
\hline Dyslipidemia & $110(58.88 \%)$ \\
\hline Chronic kidney failure & $73(39 \%)$ \\
\hline Left ventricular ejection fraction & $40 \%(I Q R$ 29-60) \\
\hline \multicolumn{2}{|c|}{ Previous medication: } \\
\hline Beta-blockers & $119(64 \%)$ \\
\hline ACEI/ARB & $95(51.1 \%)$ \\
\hline Aldosterone antagonists & $59(31.7 \%)$ \\
\hline $\begin{array}{l}\text { Blood pressure at admission } \\
{[\mathrm{mm} \mathrm{Hg}]}\end{array}$ & 125 (IQR 110-141) \\
\hline $\begin{array}{l}\text { Low cardiac output syndrome/ } \\
\text { /shock at admission }\end{array}$ & $16(8.6 \%)$ \\
\hline HVRAF at admission & $31(16.6 \%)$ \\
\hline \multicolumn{2}{|l|}{ Laboratory tests: } \\
\hline Glycemia [mg/dL] & 144 (IQR 117-192) \\
\hline Hemoglobin [mg/dL] & 10.6 (IQR 9-12) \\
\hline Creatinine $[\mathrm{mg} / \mathrm{dL}]$ & 1.8 (IQR 1.4-2.5) \\
\hline Troponin [ng/L] & 42 (IQR 24-81) \\
\hline Hospital stay [days] & 5 (IOR 4-8) \\
\hline Use of inotropic agents & $19(10.2 \%)$ \\
\hline Use of intra-aortic balloon pump & $4(2.1 \%)$ \\
\hline Use of mechanical ventilation & $5(2.7 \%)$ \\
\hline Use of hemodialysis & $9(4.8 \%)$ \\
\hline
\end{tabular}

ACEI/ARB - angiotensin-converting enzyme inhibitors/angiotensin II receptor blockers; HVRAF - high ventricle rate atrial fibrillation; $\mathrm{IQR}$ - interquartile range; SD — standard deviation

The principal characteristics of the population are described in Table 1 . Ischemic etiology was present in about $50 \%$ of patients with $\mathrm{HF}$, and $53.8 \%$ ( $\mathrm{n}=100,1$ missing datum) had an $\mathrm{EF}<45 \%$. In $93 \%$ of patients $(n=174)$, hs-cTn was above the maximal normal value (14 $\mathrm{ng} / \mathrm{L})$; median troponin level at admission was $42 \mathrm{ng} / \mathrm{L}$ (IQR 24-81). The most common clinical presentation was systemic congestion $(\mathrm{n}=144,77 \%)$. Patients were hospitalized for a median of 5 days (95\% CI 4-8) and 12 patients $(6.4 \%)$ died during hospitalization.

The history of chronic kidney failure was associated with elevated troponin values $(51 \mathrm{ng} / \mathrm{L}$, IQR $33-72$ vs. $31 \mathrm{ng} / \mathrm{L}, \mathrm{IQR} 22-57$, respectively, $\mathrm{p}=0.002$ ). In the same way, patients with ischemic

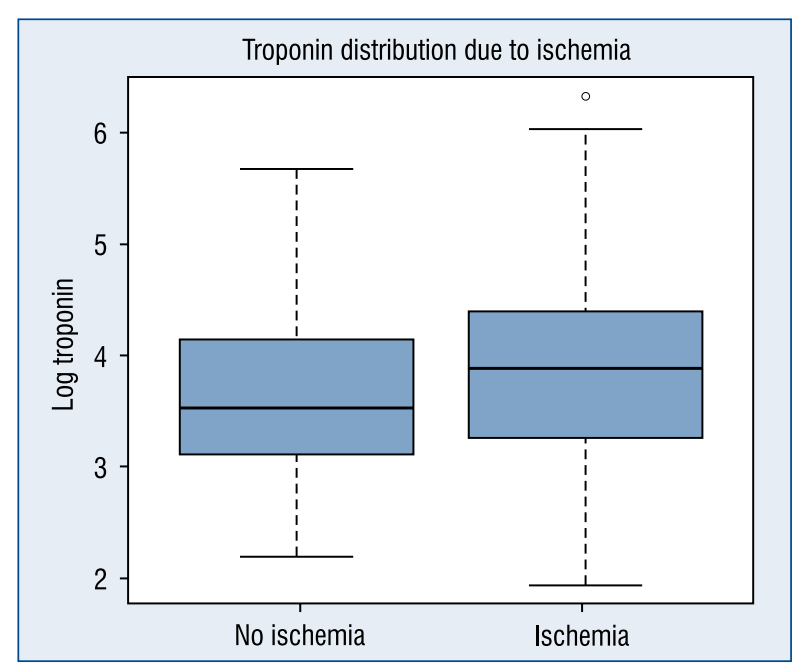

Figure 1. Statistically significant differences of troponin values according to etiology $(p=0.039)$.

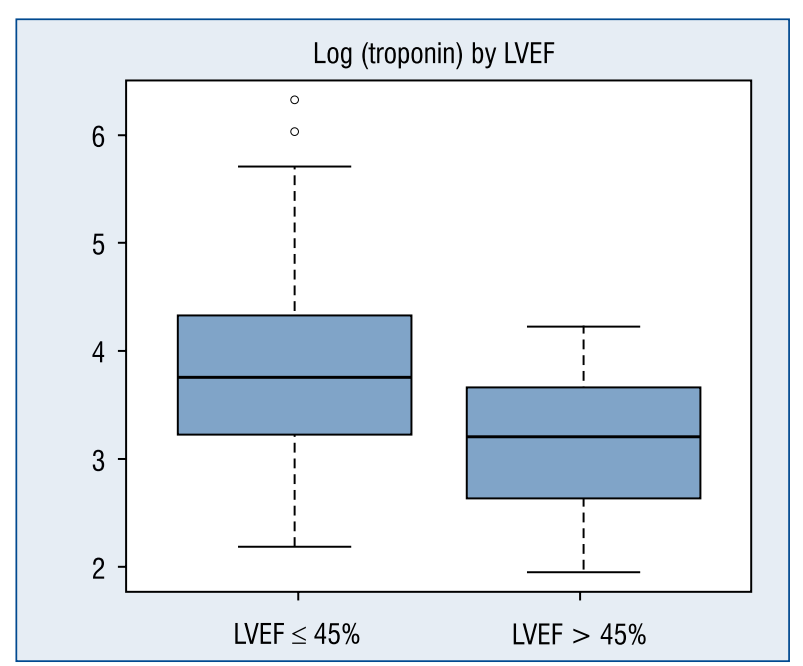

Figure 2. Distribution of troponin values according to left ventricular ejection fraction (LVEF) $>$ or $\leq 45 \%$ ( $p=0.0004)$.

etiology had significantly higher troponin values compared with those without ischemic etiology (49 ng/L, IQR 25-75: $26-82$ vs. $34 \mathrm{U} / \mathrm{L}, \mathrm{IQR}$ 25-75: 22-62, respectively, $\mathrm{p}=0.021$ ) (Fig. 1). There were no significant differences in sex, age or blood pressure at admission. Patients with left ventricular (LV) $\mathrm{EF} \leq 45 \%$ had higher troponin values (43 ng/L, IQR 25-67 vs. 25 ng/L, IQR 16-30, $\mathrm{p}=0.0004$ ) (Fig. 2).

Patients with low cardiac output syndrome or cardiogenic shock at admission had higher troponin levels compared with those with less severe clini- 
cal presentations (73 ng/L, IQR 53-113 vs. $39 \mathrm{ng} / \mathrm{L}$, IQR 24-58, $\mathrm{p}=0.004$ ).

During hospitalization, those who required inotropic agents presented higher troponin values at admission ( $76 \mathrm{ng} / \mathrm{L}$, IQR $50-107$ vs. $39 \mathrm{ng} / \mathrm{L}, \mathrm{IQR}$ $23-58, \mathrm{p}=0.002$ ), and troponin values at admission were also higher in those requiring complex therapies as intra-aortic balloon pump, mechanical ventilation or hemodialysis (86 ng/L, IQR 57-131 vs. $40 \mathrm{ng} / \mathrm{L}, \mathrm{IQR} 23-58, \mathrm{p}=0.002$ ). Median hospitalization stay was longer in patients with elevated troponin values (> $42 \mathrm{ng} / \mathrm{L}$ ) (5 days, IQR 3-8 vs. 6 days, IQR $4-9, \mathrm{p}=0.03)$.

\section{Follow-up at 90 days}

Six patients were lost during follow-up, $28(15.5 \%)$ patients died and 27 rehospitalizations occurred (55 events). Median survival was 81 days for patients with hs-cTn $<42 \mathrm{ng} / \mathrm{L}$ (95\% CI 77-85.9) and 73.6 days for patients with hs-Tn $>42 \mathrm{ng} / \mathrm{L}$ (95\% CI 67.8-79.5); this difference had statistical significant (Cox-Mantel test $\mathrm{p}=0.018$ ) (Fig. 3). The probability of not having an event at 90 days was estimated in 0.775 (95\% CI $0.689-0.862)$ for patients with hs-cTn $<42 \mathrm{ng} / \mathrm{L}$ and 0.615 (95\% CI $0.515-0.715)$ for those with higher values.

In patients with hs-cTn $>42 \mathrm{ng} / \mathrm{L}$, the risk of death or rehospitalization at 90 days was greater than in patients with low blood pressure at admission, low cardiac output syndrome or cardiogenic shock, LV EF $\leq 45 \%$ and use of inotropic agents (Table 2). Multivariate analysis showed that the use of inotropic agents was the unique variable that predicted increased risk of death or rehospitalizations at 90 days (Table 3 ).

\section{Discussion}

Cardiac troponin constitutes a well established diagnostic tool in ACS [1]. Troponins are released

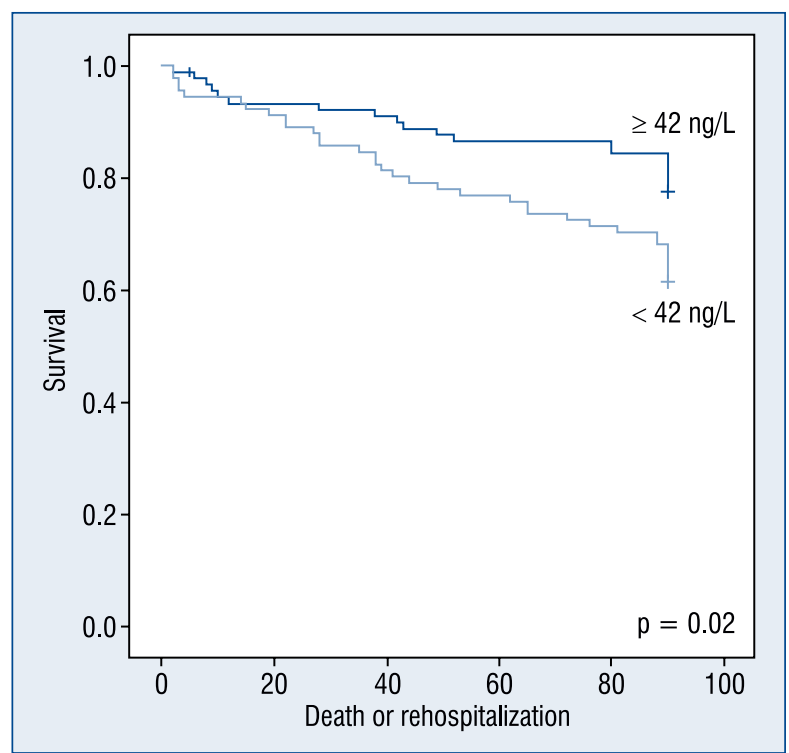

Figure 3. Survival and risk of hospitalizations according to troponin level (categorical variable $42 \mathrm{ng} / \mathrm{L}$ ).

into the circulation as an expression of myocardial necrosis and have been widely investigated in $\mathrm{HF}$ populations [3, 4]. As in other series, elevated hscTn levels were almost constant in the population studied $[5,6]$. The progressive loss of myocytes in $\mathrm{HF}$ is greater during exacerbations due to increased capillary pulmonary pressure, lower coronary perfusion pressure, greater release of catecholamines, abnormal calcium metabolism, greater oxidative stress, and inflammation, etc. This produces higher levels of circulating troponin as an expression of myocardial damage $[7,8]$. Up to the present time, the published studies have evaluated conventional troponin, and most of them demonstrated elevated troponin levels independently of the etiology of HF. We believe that our study is the first one to demonstrate that subjects with coronary artery

Table 2. Variables associated with greater risk of rehospitalization or death at 90 days.

\begin{tabular}{lcccc}
\hline Variable & $\mathbf{P}$ & Hazard ratio (HR) & \multicolumn{2}{c}{$\mathbf{9 5 \% \text { confidence interval for HR }}$} \\
\cline { 4 - 5 } & & & Lower limit & Upper limit \\
\hline Median troponin 42 ng/L & 0.021 & 1.911 & 1.1 & 3.3 \\
Inotropic agent & $<10^{-6}$ & 5.45 & 2.98 & 9.96 \\
Interquartile range & 0.155 & 1.468 & 0.87 & 2.5 \\
Left ventricular ejection fraction $\leq 45 \%$ & 0.005 & 2.37 & 1.31 & 4.3 \\
Systolic blood pressure at admission & 0.002 & 0.983 & 0.97 & 0.99 \\
Age & 0.37 & 0.990 & 0.97 & 1.01 \\
Low cardiac output/shock & 0.0001 & 4.5 & 2.3 & 8.8 \\
\hline
\end{tabular}


Table 3. Multivariate analysis to predict risk of hospitalization or death at 90 days.

\begin{tabular}{lcccc}
\hline Variable & P & Hazard ratio (HR) & \multicolumn{2}{c}{ 95\% confidence interval for HR } \\
\cline { 4 - 5 } & & & Lower limit & Upper limit \\
\hline Systolic blood pressure at admission & 0.073 & 0.99 & 0.979 & 1.001 \\
Troponin > 42 ng/L & 0.077 & 1.678 & 0.948 & 2.977 \\
Left ventricular ejection fraction $\leq 45 \%$ & 0.136 & 1.628 & 0.858 & 3.088 \\
Inotropic agents & 0.008 & 2.926 & 1.318 & 6.498 \\
Low cardiac output/shock & 0.794 & 1.033 & 0.808 & 1.321 \\
\hline
\end{tabular}

disease have greater hs-cTn levels than those with other etiologies. With the exception of the study published by Peacock et al. [9], the other authors have not been able to demonstrate a statistically significant difference according to the etiology [10, 11]. A possible explanation is that the patients with severe coronary stenoses may be more liable to the stress produced by an acute episode and, thus, have higher troponin levels. In this way, it may be interesting to determine the potential usefulness of the biomarker to identify patients with unknown etiology of HF.

Elevation of hs-cTn was associated with the most severe clinical profile; these patients were much more likely to be admitted with low cardiac output syndrome or cardiogenic shock or to require inotropic agents and complex therapies as mechanical ventilation, intra-aortic balloon pump or hemodialysis. Patients with higher troponin levels were those with ventricular dysfunction and required longer hospital stay. Up to now, the usefulness of high sensitivity cardiac troponin to identify patients with the most severe clinical profile, worse outcome during hospitalization or LV dysfunction has not been evaluated yet. Horwich et al. [10] demonstrated that in patients with ventricular dysfunction referred for heart transplantation, elevated cardiac troponin I was associated with impaired hemodynamics: higher pulmonary capillary wedge pressure, lower cardiac index and progressive $\mathrm{LV}$ dysfunction. The findings reported by Peacock et al. [9] in the ADHERE study which evaluated the role of standard troponin were very similar to those of our study: patients with higher troponin levels at admission presented lower blood pressure, had ventricular dysfunction, used more resources (intra-aortic balloon pump and mechanical ventilation) and hospital stay was longer. These findings suggest that abnormally high troponin levels at admission can be a useful parameter to identify patients with LV dysfunction, worse outcomes and greater use of resources during hospitalization. These patients could be admitted to critical care areas in order to implement the necessary treatment. On the contrary, patients with lower levels at admission could be considered of low risk and thus admitted to intermediate care units.

During follow-up, high troponin levels, low blood pressure at admission, low cardiac output syndrome or cardiogenic shock, LV dysfunction, use of inotropic agents and complex therapies were associated with greater risk of mortality or rehospitalizations. All these variables have been associated with worse outcome in previous publications. The novel information provided by this study is the integration of predictors of adverse outcomes with troponin levels. As expected, the use of inotropic agents was a stronger predictor of events during follow-up compared with troponin levels. This finding may be explained because the inotropic requirements indicate a very high risk population where troponin elevation is not only an expression of myocardium damage due to the background disease but also because the inotropic drugs cause cellular necrosis with even more troponin increscent. Interestingly, in those subjects with less severe clinical presentations, high troponin levels at admission are useful in identifying the populations with higher risk of rehospitalizations or death which should undergo strict follow-up after discharge. High hs-cTn levels also help to identify patients requiring hospitalization in critical care areas and invasive therapies.

The absence of serial troponin determinations during hospitalization is one of the limitations of this study, as we could not evaluate the biological behavior of the biomarker and the modifications produced by the treatments. As we did not use other biomarkers already studied in HF, as conventional troponin or natriuretic peptides, we could not make comparisons between them to identify the best marker for patients' stratification. 


\section{Conclusions}

Elevation of hs-cTn is a finding almost constant in patients with decompensated HF. In subjects with higher troponin levels, coronary artery disease and LV dysfunction are very frequent. The use of hs-cTn for risk stratification at admission helps to identify populations with poor outcome during hospitalization and higher risk of death or rehospitalizations during follow-up who will require rapid implementation of an aggressive treatment.

\section{Conflict of interest: None declared}

\section{References}

1. Meier MA, Al-Badr WH, Cooper JV et al. The new definition of myocardial infarction: Diagnostic and prognostic implications in patients with acute coronary syndromes. Arch Intern Med, 2002; 162: 1585-1589.

2. Gaggin HK, Januzzi Jr JL. Biomarkers and diagnostics in heart failure. Biochim Biophys Acta, 2013; 1832: 2442-2450.

3. Kalogeropoulos AP, Georgiopoulou VV, Butler J. Clinical adoption of prognostic biomarkers: The case for heart failure. Prog Cardiovasc Dis, 2012; 55: 3-13.
4. Bjurman C, Holmström A, Petzold M, Hammarsten O, Fu ML. Assessment of a multi-marker risk score for predicting causespecific mortality at three years in older patients with heart failure and reduced ejection fraction. Cardiol J, 2015; 22: 31-36.

5. Pascual-Figal DA, Casas T, Ordoñez Llanoz J et al. High sensitive troponin T for risk stratification of acute destabilized heart failure. Am Heart J, 2012; 163: 1002-1010.

6. Latini R, Masson S, Anand IS et al.; Val-HeFT Investigators. Prognostic value of very low plasma concentration of troponin T in patients with stable chronic heart failure. Circulation, 2007; 116: $1242-1249$.

7. Apple FS, Wu AHB, Jaffe AS. European Society of Cardiology and American College of Cardiology guidelines for redefinition of myocardial infarction: How to use existing assays clinically and for clinical trials. Am Heart J, 2002; 144: 981-986.

8. Kociol RD, Pang PS, Gheorghiade M, Fonarow GC, O'Connor CM, Felker GM. Troponin elevation in acute heart failure prevalence, mechanisms, and clinical implications. J Am Coll Cardiol, 2010; 56: 1071-1078.

9. Peacock WF, De Marco T, Fonarow GC et al. Cardiac troponin and outcome in acute heart failure. N Engl J Med, 2008; 358: 2117-2126.

10. Horwich TB, Patel J, MacLellan WR, Fonarow GC. Cardiac troponin I is associated with impaired hemodynamics, progressive left ventricular dysfunction, and increased mortality rates in advanced heart failure. Circulation, 2003; 108: 833-838.

11. Felker GM, Hasselblad V, Tang WHW et al. Troponin I in acute descompensated heart failure: Insgihts from the ASCEND: HF study. Eur Heart J, 2012; 14: 1257-1264. 